\title{
Oclusión de la vena central de la retina en paciente con diabetes mellitus tipo 2
}

Reporte de un caso

Medina-Gallardo Diana Florencia*, Contreras-Rodríguez Cuauhtémoc*, Torres-Bernal Luis Fernando**, Rivera-Barragán Virgilio***, Ramírez-Ramírez Carlos Antonio*

\section{Resumen}

- La oclusión vascular de la retina (OVR) es la segunda enfer-

- medad vascular más frecuente. Se clasifica en dos tipos: oclu-

- sión de una rama de la vena retiniana (ORVR) y oclusión de

- la vena central de la retina (OVCR). En la ORVR, la afección

- está confinada a un cuadrante de la retina, mientras que en

- la OVCR, se afecta los cuatro cuadrantes. La oclusión venosa

- central de la retina es clasificada ya sea como isquémica (no

- perfundida) o no isquémica (perfundida). Es diagnosticada

- con la ayuda de la fluorangiografía como complemento a

- la clínica. El objetivo de este trabajo fue revisar el concepto

- de oclusión de la vena central de la retina, el abordaje diag-

- nóstico y terapéutico de un caso clínico. UXMÉDICA, AÑO 13,

- NÚMERO 37, SEPT 2017 ABR 2018 PP 37-42

Palabras clave: oclusión de la vena central de la retina, OVCR.

\section{Abstract}

Retinal vascular occlusion (RVO) is the second most frequent vascular disease. It is classified into two types: branch retinal vein occlusion (BRVO) and central retinal vein occlusion (CRVO). In BRVO, the condition is confined to one quadrant of the retina, while in the CRVO, the four quadrants are affected. Central retinal vein occlusion is classified either as ischemic (non-perfused) or non-ischemic (perfused). It is diagnosed with the help of fluorescein angiography as a complement to the clinical evaluation. The objective of this work was to review the concept of central retinal vein occlusion, the diagnostic and therapeutic approach of the clinial CaSe. LUXMÉDICA, AÑO 13, NÚMERO 37, SEPT 2017 ABR 2018 PP 37-42

Keywords: central retinal vein occlusion, CRVO.

\section{Introducción}

La oclusión vascular de la retina (OVR) es la segunda enfermedad vascular más frecuente, es precedida sólo por la retinopatía diabética y representa la quinta causa de ceguera. ${ }^{1}$ Se clasifica en dos tipos: oclusión de una rama de la vena retiniana (ORVR) y oclusión de la vena central de la retina (OVCR), afectando a 50 de cada 100,000 pacientes. ${ }^{2}$ En la ORVR la afección está confinada a un cuadrante de la retina, mientras que en la OVCR se afecta los cuatro cuadrantes. ${ }^{3}$

* $\quad$ Estudiante de séptimo semestre de la carrera de Médico Cirujano de la Universidad Autónoma de Aguascalientes

** Médico oftalmólogo en INOVA visión quirúrgica y docente en la Universidad Autónoma de Aguascalientes.

*** Cirujano laparoscopista e investigador de la Universidad Autónoma de Aguascalientes

Fecha de recibido: 15 de febrero 2018

Fecha de aceptación: 5 de marzo 2018

Correspondencia: Dr Virgilio Rivera Barragán. Departamento de Cirugía. Edificio 107 planta baja. Centro de Ciencias de la Salud. Campus Universitario. Universidad Autónoma de Aguascalientes. Avenida Universidad 940. Código postal 20131. Aguascalientes, Ags., México. Teléfono 01 (449) 9108443. Correo electrónico dr.virgilio.rivera@gmail.com 
La hipertensión arterial sistémica y/o dislipidemias son enfermedades en común preexistentes entre ambos. ${ }^{3}$ La OVCR se ve, principalmente, en personas con una media de edad de 50 años. El etilismo y la diabetes mellitus son los factores predisponentes que duplican el riesgo a contraer la enfermedad, sumándole la importante asociación con glaucoma de ángulo abierto de forma crónica y el incremento en la actividad física, todo viéndose en el $75 \%$ de los casos de OVCR. ${ }^{1,4}$

La enfermedad tiene como origen genético la resistencia a la proteína $C$ activada, ya que hay una mutación del factor $V$ de Leiden. Este factor es la causa genética más común para desarrollar trombosis venosa. Su presencia retrasa la recirculación e isquemia, resultando en un elemento de alto riesgo de la complicación neovascular en OVCR. Deficiencias de proteínas de anticoagulación, como son la antitrombina III, proteína $C$ y proteína $S$ y altos niveles de factor VII, lipoproteína (a), factor VIII, receptor endotelial de proteína $\mathrm{C}$ e hiperhomocisteinemia, son reportados como otros posibles factores a la formación de trombos en la vena central de la retina que, finalmente, provocará una disminución de la luz intravascular ocluyéndola. ${ }^{4}$

Green y cols. encontraron que el probable mecanismo fisiopatológico, puede ser la compresión de la vena por la arteria a nivel de la lámina cribosa que produce turbulencia, agregación plaquetaria y formación de trombos. Las plaquetas y el endotelio vascular generan derivados de las prostaglandinas que presentan efectos farmacológicos opuestos. Las plaquetas en el sitio afectado liberan tromboxano A2, que actúa como agregante plaquetario y vasoconstrictor. ${ }^{1}$

El edema macular, complicación de la oclusión venosa central de la retina, unilateralmente ocasiona que la agudeza visual se vea afectada, de manera indolora, ${ }^{1,2}$ con un $15 \%$ de contraerla de manera bilateral. 2,3 Su origen puede ser de tres distintas maneras: 1) compresión externa sobre la vena, 2) enfermedad venosa primaria y 3) una trombosis. El mecanismo probable de la oclusión puede ser por compresión de la arteria a nivel de la lámina cribosa provocando turbulencia, agregación plaquetaria y finalmente la formación de trombos. ${ }^{1}$

La oclusión venosa central de la retina es clasificada como isquémica (no perfundida) o no isquémica (perfundida). ${ }^{1,4}$ La fluorangiografía se solicita como complemento de los hallazgos clínicos. Una de las principales características clínicas para diferenciarlas, empezando con la no perfundida, es que la agudeza visual es $\leq 20 / 200$, presencia de exudados blandos abundantes, defecto pupilar aferente, que es un buen predictor en el riesgo de complicaciones neovasculares, mayor de 10 áreas de no perfusión capilar con ayuda de la fluorangiografía y hemorragias abundantes. ${ }^{1,3,4}$ La OVCR es una notable causa precedente para formar neovascularización del iris y glaucoma neovascular como una complicación de ésta en 


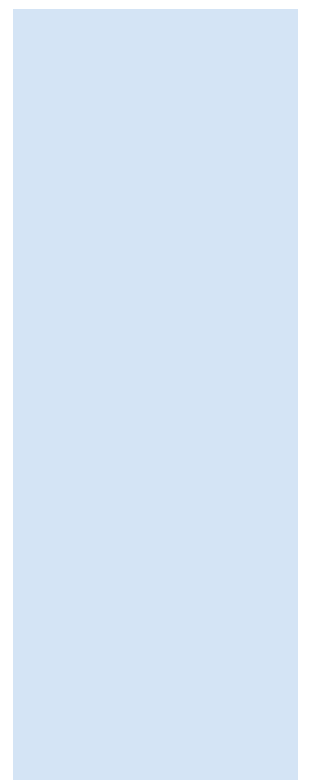

un $40 \%$ a $85 \%$ en la forma isquémica de la enfermedad, y sólo $5 \%$ en la no isquémica. ${ }^{1,4}$ La no isquémica o perfundida tiene como característica clínica una agudeza visual $\geq 20 / 200$, sin presencia de defecto pupilar aferente, escasas hemorragias, así como pocos exudados húmedos y buena perfusión capilar.

En los estudios de población de mediana edad y los adultos mayores, la prevalencia de la OVR osciló entre 0,7\% al 1,6\%. La incidencia y prevalencia de la OVR aumenta con la edad y no tiene predilección por el sexo. En los cuatro principales grupos étnicoraciales en los Estados Unidos (blancos, afro- americanos, hispanos y chinos), la prevalencia es similar. En un estudio realizado en nuestro país, se observó que de la OVR, 43.2\% correspondió a OVCR y $56.8 \%$ a ORVR, con un rango de edad de 26 a 86 años, siendo el promedio de 64.8 años, con una DE de 11.32. La HAS estuvo presente como antecedente en $67.6 \%$ de los casos, el grupo de edad más afectado en mujeres fue de 75 a 79 años y en hombres de 80 a 84 años. ${ }^{5}$

\section{Presentación del caso clínico}

Masculino de 41 años de edad, originario del municipio de Jesús María en el estado de Aguascalientes. Con antecedente de diabetes mellitus tipo 2 de cinco años de evolución, controlado con metformina y glibenclamida. Además con hipertensión arterial de tres años de evolución manejada con metoprolol. Inició su padecimiento con disminución de la agudeza visual en ojo izquierdo, indolora, por lo que acude a valoración oftalmológica, donde se observa una agudeza visual sin corrección de 20/60 en ojo derecho y 20/70 en ojo izquierdo.

A la exploración del segmento anterior con lámpara de hendidura, se encuentra opacidades corticales posteriores periféricas en ambos ojos compatibles con diagnóstico de cataratas (figura 1). En fondo de ojo derecho, se observan hemorragias en los cuatro cuadrantes de la retina, microaneurismas y exudados duros. En la fluorangiografia, se observan áreas hipofluorescentes con pérdida de la trama vascular (figuras 2 a y 2 b). En la tomografía de coherencia óptica, se encuentra edema papilar (figura 3).

Se inicia tratamiento con ranibizumab, mostrando mejoría de los síntomas, específicamente de la agudeza visual de ojo izquierdo sin corrección 20/60, con agujero estenopeico de 20/30 y con corrección 20/25. Se solicita seguimiento de control mensual los primeros seis meses y posteriormente anual. Se refiere al servicio de Medicina Interna para control de patologías sistémicas subyacentes.

\section{Discusión}

La oclusión de la vena central de la retina es mucho más común que la oclusión de la arteria. Siendo más común en personas mayores de 50 años. ${ }^{1,3}$
El paciente del caso clínico se presentó a valoración en una fase tardía, con disminución de la agudeza visual, hemorragias, microaneurismas y exudados en los cuatro cuadrantes de la retina, opacidades corti- 
cales en ambos ojos observadas con lámpara de hendidura, con marcado edema macular en la tomografía de coherencia óptica, zonas hipofluorescentes y pérdida de la trama vascular en la fluorangiografía. El dato clínico que resaltaba durante la evaluación inicial era la disminución de la agudeza visual sin dolor, que es una causa frecuente de consulta al oftalmólogo y que al inicio nos hizo pensar en un origen vascular.

La agudeza visual disminuida es justificada por la presencia de edema macular, el uso de la tomografía de coherencia óptica resultó de utilidad para evaluar el grado de severidad de ésta y como auxiliar diagnóstico. La búsqueda de las características en la fluorangiografía serán cambios del calibre vascular, anormalidades en la permeabilidad vascular y cierre de capilares

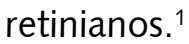

Nuevos tratamientos han sido sugeridos a través de los años dirigidos a combatir el edema macular: el desarrollo de neovascularidad y el uso de corticosteroides. ${ }^{6}$ El uso de triamcinolona intra-vítrea como corti- costeroide reduce la permeabilidad vascular y estabiliza la barrera sangre-retina. ${ }^{6,7}$ La hipoxia en células favorece la producción del factor de crecimiento endotelial vascular aumentando la permeabilidad y proliferación. $6,8,9$ Los antiangiogénicos como pegaptanib, bevacizumab, ranibizumab, aflibercept, se unen al factor de crecimiento endotelial vascular para inhibir la unión al receptor endotelial. ${ }^{8}$

El tratamiento de este paciente fue con ranibizumab administrado vía intravítrea. Este tratamiento también está indicado en el tratamiento de degeneración macular asociada a la edad, neovascularizacion coroidea y edema macular diabético. La reacciones relacionadas con la inyección intravítrea de ranibizumab incluyen endoftalmitis, desprendimiento de retina regmatógeno, catarata traumática iatrogénica y aumento de la presión intraocular en los 60 minutos siguientes a la inyección. ${ }^{10,11}$

Para el tratamiento exitoso de las oclusiones de vena central de la retina, se deben tratar las enfermedades sistémicas concomitantes como en este caso.

\section{Conclusiones}

La oclusión de la vena central de la retina es una enfermedad relativamente frecuente, observada en una media de 60 años. Existen factores de riesgo en dos terceras partes de quienes la padecen, como lo son la diabetes mellitus, hipertensión, el glaucoma de ángulo abierto, además de factores de hipercoagulabilidad que favorezcan la formación de un trombo que disminuirá la luz de la vena de la retina.

Las complicaciones de este padecimiento son también de importante diagnóstico, ya que involucran la formación de neovasos, los cuales no son de calidad, forman aneurismas y se rompen con mayor facilidad. Si bien el tratamiento farmacológico se ha visto muy favorable a combatir la angiogénesis y el edema macular, también se debe tratar la causa primaria de esto que es la descompresión de la vena de la retina mediante intervenciones quirúrgicas por láser. 

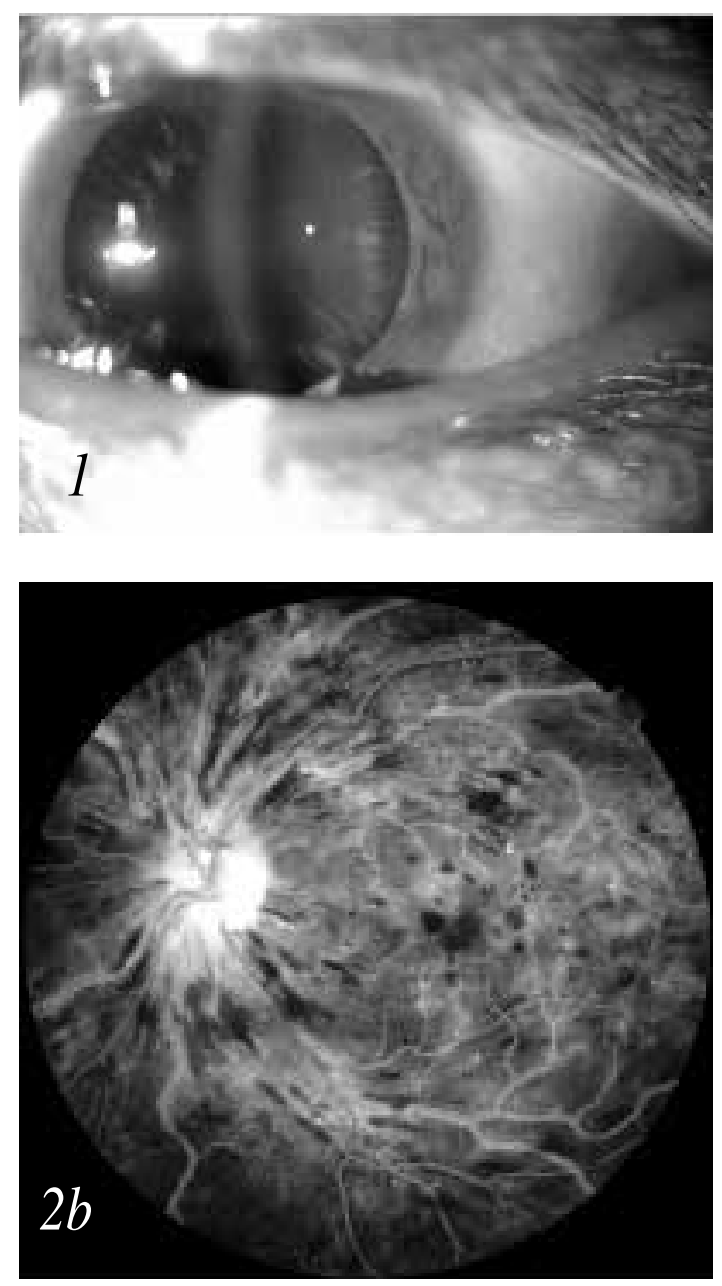

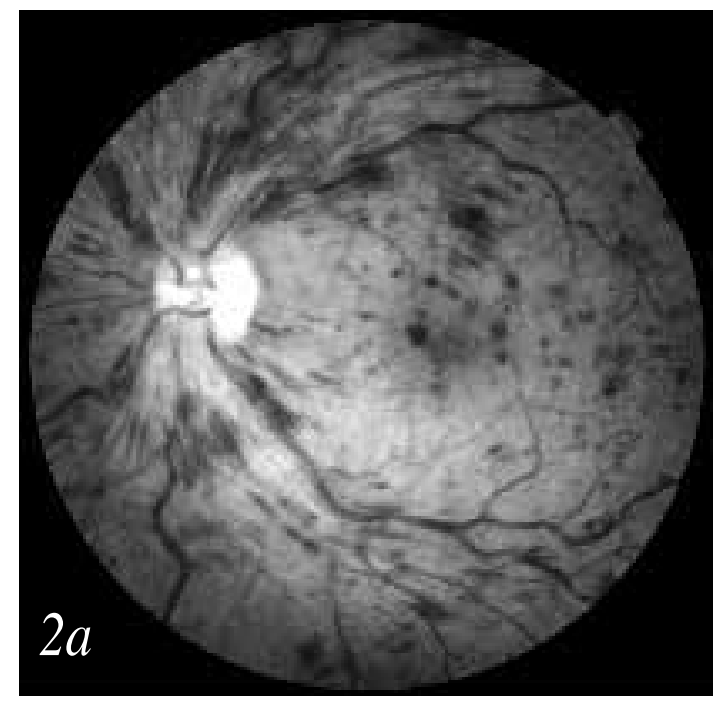

Figura 1. Ojo izquierdo de paciente con opacidades corticales posteriores.

Figura $2 a$ y $2 b$. Fluorangiografía de ojo izquierdo en paciente con oclusión de la vena central de la retina en la que se observan hemorragias, microaneurismas y exudados duros en los cuatro cuadrantes.

\section{Análisis de Cambio de Retina Map}

Izquierda / OS
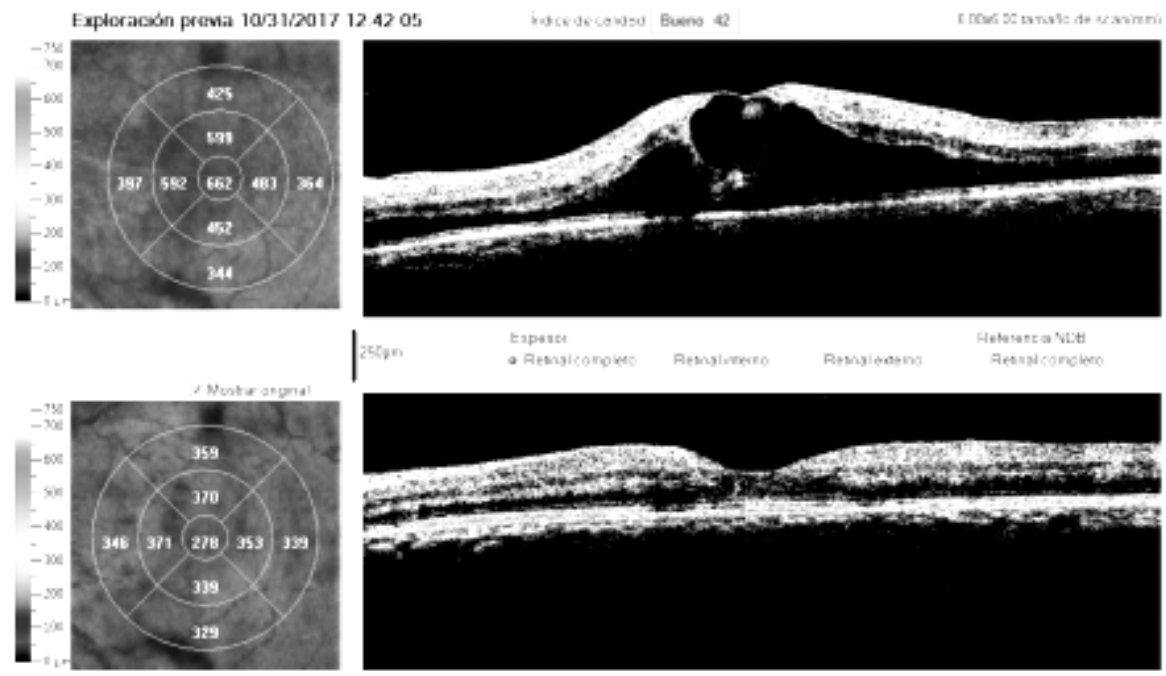

Exroracoón rocente 11/06r20 1/ 10:31 00

hiscedecowsol Bum 80

Figura 3. Tomografía de coherencia óptica de ojo izquierdo en paciente con oclusión de la vena central de la retina en la que se observa edema macular. 


\section{Bibliografía}

1. Rodríguez-Loaiza JL, Graue-Wiechers F. Oclusión de la vena central de la retina. Rev Mex Oftalmol. 2003;77:83-87.

2. Cruz Niesvaara D, Ibero Villa JL, Leiva Aranda I, Blanco López B. Oclusión de la vena central de la retina. Semergen. 2006;32(9):468-469.

3. Kida T. Mystery of retinal vein occlusion: Vasoactivity of the vein and posible involvement of endothelin-1. Biomed Res Int. 2017;2107:1-16.

4. Berker N, Batman C. Surgical treatment of central retinal vein occlusion. Acta Ophthalmol. 2008;86:245252.

5. IMSS. Diagnóstico y tratamiento de la oclusión venosa de retina. México D.F. 2013; 1-51.

6. Querques G, Triolo G, Casalino G, et.al. Retinal Venous Occlusions: Diagnosis and Choise of Treatments. Ophthalmic Res. 2013;49:215-222.
7. Adami Lucatto LF, Magalhães Junior $O$, Prazeres $J M B$, et.al. Incidence of anterior segment neovascularization during intravitreal treatment for macula edema secundary to central retinal vein occlusion. Arq Bras Oftalmol. 2017;80(2):97-103.

8. Rhoades W, Dickson D, Nguyen QD, Do DV. Management of macular edema due to central retinal vein occlusion - The rone of aflibercept. Taiwan J Ophthalmology. 2017;7(2):70-76.

9. Quiroz Mercado $H$, Velez Montoya R, Fromow Guerra J, Guerrero Naranjo JL, Moreno Paramo D, Morales Canton V. Terapia antiangiogénica ocular: experiencia clínica en México. Gac Med Mex. 2008;144(3):245-253.

10. Lucentis. INNA- Ranibizumab. Europa https:// ec.europa.eu $>$ anx 136324 es

11. Cruz N, Ibero V, Leiva I, Blanco B. Oclusión de la vena central de la retina. Semergen. 2006;32 (9): 468-469. 